\title{
Construcción de cuentos: ¿Qué pueden aportar al desarrollo socioemocional inclusivo?*
}

\author{
How can the creation of stories contribute to an inclusive socio-emotional development?
}

\author{
Pilar Álamos, ${ }^{a}$ Oriana Cifuentes, ${ }^{a b}$ Neva Milicic,${ }^{a c}$ Marcelo Pizarro, ${ }^{d}$ \\ Ricardo Rosas, ${ }^{\text {de }}$ Denisse Ulloa, ${ }^{\text {af }}$ Soledad Véliz ${ }^{d g}$ \\ aPontificia Universidad Católica de Chile, Facultad de Ciencias Sociales, VALORAS UC \\ Correo electrónico: pmalamos@uc.cl

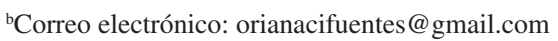 \\ ${ }^{c}$ Correo electrónico: nmilicic@uc.cl \\ 'Pontificia Universidad Católica de Chile, Facultad de Ciencias Sociales, CEDETI UC \\ Correo electrónico: marcelopizarro@uc.cl \\ ${ }^{\mathrm{e}}$ Correo electrónico: rrosas@uc.cl \\ ${ }^{\mathrm{f} C}$ Correo electrónico: denisseulloap@ gmail.com \\ gCorreo electrónico: sdveliz@uc.cl
}

\section{RESUMEN}

Este artículo da cuenta de la implementación de un taller piloto para la promoción del desarrollo socioemocional de estudiantes de quinto básico a través de la creación de narrativas con contenido inclusivo. El taller consistió en 7 sesiones de 90 minutos; en cada sesión se realizaron actividades de trabajo colaborativo orientadas a la creación de cuentos. Se utilizó una metodología en dos fases: desarrollo de contenidos/herramientas de evaluación e implementación del taller. Se reportan mejores resultados en pruebas de autoestima tanto en niños identificados como tímidos así como en niños identificados como disruptivos por sus profesores. Los análisis no son concluyentes respecto del impacto global del taller sobre el desarrollo socioemocional. Es posible destacar que tanto los niños como los profesores reportaron altos niveles de satisfacción con la experiencia. Se discute el potencial del taller como experiencia significativa en el desarrollo socioemocional.

Palabras clave: cuentos, aprendizaje socioemocional, inclusión.

\begin{abstract}
The present article refers to the implementation of a pilot workshop for the promotion of the socio-emotional development of fifth grade students through the creation of narratives with an inclusive content. The workshop comprised 7 sessions lasting 90 minutes, each of which involved collaborative activities oriented towards story creation. A two-phase methodology was used: contents and evaluation tools were developed first, with the workshop being implemented afterwards. Children identified as shy or disruptive by their teachers scored higher in self-esteem tests after the workshop. The overall analyses are not conclusive regarding the global impact of the workshop on socio-emotional development. It should be pointed out that both children and teachers who participated reported high satisfaction levels with the experience. The article also presents a discussion on the potential of the workshop as a significant socio-emotional development experience.
\end{abstract}

Key words: stories, socio-emotional learning, inclusion.

\footnotetext{
Investigación financiada por el Ministerio de Educación de Chile a través del proyecto FONIDE F811341.
} 


\section{INTRODUCCIÓN}

En los últimos años, la calidad de la educación escolar ha sido uno de los temas de mayor debate público. Existe consenso de que esta debe tender hacia un desarrollo integral del alumno, que comprenda no solo la dimensión académica, sino también otras áreas del desarrollo humano (Agencia Calidad Educación, s/f; Delors, 1997; Morín, 1999). El presente trabajo busca aportar en la integración de "lo socioemocional" al aprendizaje académico, bajo la convicción de que el desarrollo socioemocional es esencial no solo para el bienestar psicológico y social de los individuos, sino también para su desempeño académico (Berger et al., 2014; Hruby et al., 2011; Ripley \& Simpson, 2007; Shonkoff \& Phillips, 2000; Thompson, 2010).

Bajo esta misma convicción, varios trabajos se han enfocado en el desarrollo de programas para el aprendizaje de competencias socioemocionales, como "New Beginnings", "Promoting Alternative Thinking Skills" o "Social and Emotional Aspects of Learning", entre otros. Dichos programas corresponden a intervenciones universales que enfatizan la enseñanza explícita de un currículum socioemocional y suelen aplicarse durante todo el año escolar. Sus resultados han dado cuenta de un impacto positivo en diversos indicadores del desarrollo infanto-juvenil (Durlak et al., 2010), por lo que han aportado evidencia en favor de la inclusión de la dimensión socioemocional en el contexto escolar. En esta línea, el llamado actual desde la investigación y políticas públicas es no solo a enseñar competencias socioemocionales de manera aislada, sino también a integrarlas al currículum académico y a las prácticas docentes cotidianas (Jones \& Bouffard, 2012). Es por eso que el objetivo general de este estudio es desarrollar, implementar y evaluar un taller que permita integrar el aprendizaje de competencias socioemocionales al currículum de lenguaje de 5 to básico.

La opción por la asignatura de lenguaje se sustentó en que la literatura infantil ha sido descrita como un medio privilegiado para la alfabetización emocional, en tanto el mundo emocional y social aparece como una parte integral de los cuentos infantiles. Por ejemplo, un estudio de 90 libros para niños halló que las referencias a emociones o eventos sociales - a través de las palabras, expresiones, imágenes y situaciones presentadas en los librosocurren en promedio cada tres oraciones (Dyer, Shatz \& Wellman, 2000). Asimismo, el uso de cuentos en forma terapéutica (Santagostino, 1997), la lectura de libros-álbum sobre migración para intencionar la acogida de estudiantes inmigrantes (Colomer, 2012) y el trabajo conjunto en lenguaje y resolución de conflictos (Brown et al., 2010) constituyen experiencias de intervención que inspiran la vinculación de la dimensión socioemocional con objetivos de aprendizaje propios de la asignatura de lenguaje.

Pese a estos esfuerzos por utilizar literatura infanto-juvenil como puente para promover el desarrollo socio-emocional, una revisión de Sanjuán (2014) acerca de la educación literaria desde los factores emocionales concluye que, más allá de los planteamientos teóricos, son todavía muy escasas las concreciones metodológicas que sustentan la lectura en las aulas como una experiencia vital que promueva el desarrollo no solo cognitivo de los estudiantes, sino también emocional. En otras palabras, la operacionalización de este vínculo entre las dimensiones cognitiva y socio-emocional sigue siendo un desafío para el campo educativo.

Una de los principales desafíos de integrar perspectivas socioemocionales al trabajo escolar es el grado de ambigüedad y confusión conceptual del concepto de aprendizaje socioemocional (Hoffmann, 2009; Humphrey, 2013). Por ello, cabe destacar que en este 
estudio se utilizó como base teórica el concepto de alfabetización emocional, definido como la competencia de reconocer adecuadamente las propias emociones y las emociones expresadas por otros, es decir, adquirir conocimiento emocional (Bailey, Denham \& Curby, 2013; Riquelme \& Munita, 2011). Se utilizó el modelo de alfabetización emocional de Antidote (2003), quien define tres dimensiones:

- Desarrollo de un lenguaje emocional: refiere a la capacidad de poner en palabras las propias experiencias emocionales.

- Capacidad de reflexionar acerca de las propias emociones: alude al contacto con las propias experiencias emocionales, a ser capaz de mirarlas y a emitir un juicio acerca de ellas.

- Demostrar interés por las emociones de otros: corresponde al componente interaccional de la alfabetización emocional, es decir, su aplicación a las relaciones interpersonales.

Estas dimensiones revelan la naturaleza evolutiva de "lo socioemocional" y su dimensión interaccional de construcción social y abierta al cambio, que emerge como una función entre factores internos y externos, y la interacción entre ambos. En relación con ello, la evidencia da cuenta de que los niños con una mejor comprensión emocional tienen expectativas más positivas respecto al comportamiento de sus pares, son más cooperadores y tienen un mejor auto-concepto, entre otros (Thompson, 2010).

A partir de los antecedentes teóricos y empíricos presentados se desarrolló un modelo de intervención centrado en (i) el desarrollo de un lenguaje emocional, (ii) la reflexión acerca de las propias emociones y (iii) el interés por las emociones de otros, a través del aprendizaje del proceso de escritura de cuentos en la asignatura de lenguaje. Específicamente, esta intervención o taller tuvo como objetivo general que los estudiantes desarrollen competencias socioemocionales a través de la creación colaborativa de un cuento en el tema de inclusión. La intervención se aplicó a dos cursos de 5to básico, de alrededor de 40 niños por curso que fueron divididos en 7 grupos. Se les invitó a participar en 7 sesiones, una vez a la semana, de dos horas pedagógicas (90 minutos) junto al docente de lenguaje y a dos investigadores.

\subsection{DESCRIPCIÓN DE CADA SESIÓN}

La intervención realizada consistió en un taller de 7 sesiones de 90 minutos. En cada sesión se desarrollaron actividades para la creación de cuentos con temáticas de inclusión. La metodología de este taller se basó en el trabajo colaborativo en la sala de clases y fue probada experimentalmente en cursos completos de 5to básico — 40 a 45 alumnos- divididos en grupos de entre 6 y 7 estudiantes dentro de la jornada escolar. Además, transversalmente se buscó que los estudiantes desarrollen lenguaje emocional y empatía que son consideradas competencias básicas para la inclusión. Adicionalmente, se informó a los participantes de los talleres que sus cuentos serían publicados en un sitio web.

Todas las sesiones tenían la siguiente estructura:

- Lectura de un cuento: cada sesión comenzaba con la lectura de un cuento en voz alta cuyo texto e imagen permitían sensibilizar a los estudiantes acerca del recono- 
cimiento y valoración de las diferencias entre las personas. Adicionalmente, se recordaba a los estudiantes las actividades realizadas en la sesión anterior.

- Actividad central: posterior a la lectura del cuento, cada sesión propuso una actividad central en grupo orientada a la escritura e ilustración de un elemento del cuento como los personajes, los escenarios o los finales de la historia. En todas las sesiones del taller la actividad central contempló el uso de materiales de arte para inducir y facilitar el proceso creativo.

- Cierre: al término de cada sesión se recogían los aspectos más relevantes del trabajo de los estudiantes, destacando el avance de cada grupo. Además, se adelantaban algunos aspectos que serían abordados en la siguiente sesión.

El desarrollo de las sesiones fue creado considerando distintos requerimientos en los tipos de interacción entre los estudiantes. Es posible identificar un tránsito en la forma de trabajo desde las interacciones en pareja hasta el trabajo en grupo. Este tránsito fue intencionado para favorecer distintos espacios de trabajo grupal, en el que surgieran distintos tipos y niveles de conflicto que requerían, a su vez, de distintas estrategias de resolución por parte de los estudiantes.

En la primera sesión se armaron los grupos de escritura. Estos grupos fueron creados por el profesor con el requerimiento de que hubiera distintas personalidades dentro de un mismo equipo, con el objetivo de evitar reunir dentro de un mismo grupo a estudiantes con un estilo similar, ya que podría afectar el desarrollo de los cuentos. En la segunda y quinta sesión los estudiantes tenían dos modos de participación. En el primero debían trabajar en duplas elaborando un producto determinado; en el segundo debían presentar el producto al resto del grupo e integrarlo a la historia. En las sesiones tercera, cuarta, sexta y séptima los estudiantes debían trabajar en grupo para generar los productos asociados a cada actividad.

En la Tabla 1 se indican los objetivos, actividades y componente socioemocional trabajado en cada sesión.

Tabla 1. Descripción de las sesiones

\begin{tabular}{|c|c|c|c|}
\hline & Objetivo & Actividades & Componente socioemocional \\
\hline Sesión 1 & $\begin{array}{l}\text { Introducir al taller de } \\
\text { escritura de cuentos }\end{array}$ & $\begin{array}{l}\text { Presentación del modelo de } \\
\text { trabajo en grupo } \\
\text { Armar grupos de trabajo }\end{array}$ & Reflexión emocional \\
\hline Sesión 2 & $\begin{array}{l}\text { Crear personajes } \\
\text { principales para la } \\
\text { historia }\end{array}$ & $\begin{array}{l}\text { Trabajo en parejas con guías } \\
\text { creadas para la elaboración } \\
\text { de los personajes } \\
\text { Trabajo grupal de presenta- } \\
\text { ción de los personajes crea- } \\
\text { dos por cada pareja }\end{array}$ & Vocabulario socioemocional \\
\hline Sesión 3 & Crear escenario & $\begin{array}{l}\text { Trabajo grupal para la crea- } \\
\text { ción de un mapa/escenario } \\
\text { que ubique a los personajes } \\
\text { creados en la sesión anterior }\end{array}$ & $\begin{array}{l}\text { Interés sobre las emociones } \\
\text { del otro }\end{array}$ \\
\hline
\end{tabular}




\begin{tabular}{|l|l|l|l|}
\hline Sesión 4 & $\begin{array}{l}\text { Crear el conflicto de } \\
\text { la historia }\end{array}$ & $\begin{array}{l}\text { Trabajo grupal sobre un per- } \\
\text { sonaje nuevo que se incorpo- } \\
\text { ra a la historia con un nuevo } \\
\text { conflicto }\end{array}$ & $\begin{array}{l}\text { Interés sobre las emociones } \\
\text { del otro }\end{array}$ \\
\hline Sesión 5 & $\begin{array}{l}\text { Elaborar desenlaces } \\
\text { posibles }\end{array}$ & $\begin{array}{l}\text { Elaboración en pareja de dis- } \\
\text { tintos finales a la historia } \\
\text { Elaboración grupal de un fi- } \\
\text { nal para la historia }\end{array}$ & Reflexión emocional \\
\hline Sesión 6 & Editar el cuento & $\begin{array}{l}\text { Edición grupal del cuento } \\
\text { para presentación final }\end{array}$ & $\begin{array}{l}\text { Interés sobre las emociones } \\
\text { del otro }\end{array}$ \\
\hline Sesión 7 & $\begin{array}{l}\text { Presentar el cuento a } \\
\text { los compañeros }\end{array}$ & $\begin{array}{l}\text { Lectura del cuento frente al } \\
\text { curso }\end{array}$ & Reflexión emocional \\
\hline
\end{tabular}

\section{MÉTODO}

\subsection{MUESTRA}

En la investigación del modelo participaron dos quintos básicos de dos colegios de la Región Metropolitana. La selección de los colegios fue intencionada de manera que ambos tuvieran al menos un $60 \%$ en índice de vulnerabilidad y un proyecto de integración escolar. En cada colegio se seleccionó al azar un curso experimental y otro control.

\subsection{INSTRUMENTOS}

Se aplicaron las formas A y B del CLP (Alliende, Condemarín \& Milicic 1990), el Test de Autoestima Escolar (TAE) profesor y alumno (Marchant, Haeusleer \& Torreti, 2001), la Escala de Clima Social Escolar (ECLIS) (Arón, Milicic \& Armijo, 2012), el Autorreporte de Clima Laboral Escolar (ACLE) (Arón \& Milicic, 2013) y una escala de alfabetización socioemocional creada en el marco del proyecto de investigación ${ }^{1}$. Además se preguntó a los profesores que participaron en el taller si tenían conocimientos sobre desarrollo socioemocional. Al término de los talleres se les pidió a los profesores y a los estudiantes que contestaran una encuesta de satisfacción.

Se pidió también a los profesores de los grupos experimentales que identificaran a los niños disruptivos y tímidos del curso. Otras investigaciones (Humphrey et al., 2010) han identificado que los niños identificados por los profesores como aquellos que requieren de más apoyos son los que más se benefician de intervenciones socioemocionales.

Adicionalmente a los instrumentos de evaluación cuantitativa, cada dupla de investigadores llevó una bitácora en la que registraban lo sucedido en cada sesión. Las anotaciones tenían el propósito de evaluar las actividades utilizadas, sugerir modificaciones dentro de la estructura ya propuesta y contar con un registro de la actitud del curso y de algunos estudiantes durante el desarrollo del taller.

La escala tiene 31 ítems y una confiabilidad de,847 en Alpha de Cronbach. El desarrollo de este instrumento será discutido en otra publicación. 


\section{RESULTADOS}

\subsection{ANÁLISIS CUANTITATIVOS}

\subsubsection{Descriptivos generales}

La Tabla 2 contiene un resumen de las diferencias de puntajes generales obtenidos por los cursos correspondientes a las condiciones control y experimental.

Tabla 2. Diferencia de puntajes generales

\begin{tabular}{|l|c|c|c|c|}
\hline \multicolumn{1}{|c|}{ Condición } & \multicolumn{2}{c|}{ Control } & \multicolumn{2}{c|}{ Experimental } \\
\hline & Diferencia pre-post & Sig. & Diferencia pre-post & Sig. \\
\hline TAE alumno & $-0,91$ &, 117 & $-3,5$ &, $001^{* *}$ \\
\hline TAE profesor & 8,47 &, $000^{* *}$ & 20,33 &, $000^{* *}$ \\
\hline ECLIS compañeros & $-1,69$ &, $036^{*}$ & $-1,31$ &, 068 \\
\hline ECLIS profesor & $-2,67$ &, 136 & $-5,62$ &, $000^{* *}$ \\
\hline ECLIS lugar & $-0,49$ &, 800 & $-0,74$ &, 073 \\
\hline ECLIS colegio & $-1,07$ &, 718 & $-1,21$ &, 416 \\
\hline ECLIS bullying & $-2,15$ &, $001^{* *}$ & $-1,9$ &, $001^{* *}$ \\
\hline ECLIS total & $-7,1$ &, 073 & $-8,73$ &, $001^{* *}$ \\
\hline Alfabetización & $-2,35$ &, 198 & $-2,95$ &, $018^{*}$ \\
\hline CLP percentil & $-3,76$ &, $37 *$ & $-7,09$ &, 136 \\
\hline CLP puntaje Z & $-0,13$ &, 64 & $-0,25$ &, 104 \\
\hline CLP puntaje T & $-1,34$ &, 64 & $-2,73$ &, 90 \\
\hline
\end{tabular}

Al realizar un análisis de medidas repetidas se encontraron diferencias significativas en el grupo control en todos los indicadores de CLP, ECLIS compañeros, ECLIS bullying y TAE profesor. Estos resultados indican que el grupo control obtuvo peores desempeños en CLP y en ECLIS compañeros; sin embargo, se redujo el bullying y mejoró la autoestima de los estudiantes de acuerdo a los profesores.

En el grupo experimental se encontraron diferencias significativas en los indicadores de TAE alumno, Alfabetización socioemocional, ECLIS profesores, ECLIS bullying, ECLIS total y TAE profesor. Estos resultados dan cuenta de peores desempeños en TAE alumno, alfabetización sociemocional, ECLIS profesores y ECLIS total; sin embargo, se redujo el bullying y mejoró la autoestima de los estudiantes percibida por el profesor.

Los resultados presentados previamente hacen necesario un segundo nivel de análisis en que se detallen los cambios a nivel de colegio. 


\subsubsection{Análisis pre y post implementación de los talleres entre grupos control y experi- mental al interior de ambos colegios}

En los resultados de los análisis PRE y POST del grupo control del colegio 1 se observan diferencias significativas en los indicadores de TAE ( $\mathrm{t}(32)=2,414, \mathrm{P}<0,05)$, ECLIS profesores $(\mathrm{t}(32)=2,378, \mathrm{P}<0,05)$ y ECLIS bullying $(\mathrm{t}(32)=3,721, \mathrm{P}<0,05)$. Todos estos indicadores tienen menores puntajes en la segunda aplicación. Los resultados del grupo experimental del mismo colegio dan cuenta de menores puntajes en TAE $(\mathrm{t}(39)=3,159, \mathrm{P}<0,05)$, la subescala de profesores de ECLIS $(\mathrm{t}(39)=1,377, \mathrm{P}<0,05)$ y ECLIS total $(\mathrm{t}(39)=2,193, \mathrm{P}<0,05)$.

En el grupo control del colegio 2 se observan menores resultados de CLP en la aplicación post $(\mathrm{t}(32)=5,066, \mathrm{P}<0,05)$; en el grupo experimental se observa una disminución en el desempeño en la aplicación post de CLP $(\mathrm{t}(36)=3,168, \mathrm{P}<0,05)$, las subescalas profesores $(\mathrm{t}(37)=3,460, \mathrm{P}<0,05)$ y bullying de ECLIS $(\mathrm{t}(37)=2,416, \mathrm{P}<0,05)$ y ECLIS total $(\mathrm{t}(37)$ $=2,577, \mathrm{P}<0,05)$.

\subsubsection{Análisis pre y post grupos especiales}

Se realizó una comparación de promedios con los puntajes obtenidos por los niños identificados como tímidos y disruptivos por los profesores. Debido al reducido número de sujetos, solo se compararon los resultados en términos de aumento en función de las desviaciones estándar de las pruebas.

Los estudiantes identificados como tímidos por sus profesores obtuvieron un puntaje promedio en TAE alumno y profesor en la medición PRE. En la medición post, los sujetos obtuvieron puntajes que se ubicaron una desviación estándar por sobre el promedio de la prueba. En el resto de las pruebas no se observaron variaciones iguales o superiores a una desviación estándar.

Los estudiantes identificados como disruptivos obtuvieron un puntaje promedio en TAE profesor en la evaluación PRE. En la evaluación POST, los resultados de TAE profesor de este grupo se encuentran una desviación estándar por sobre el promedio normativo de la prueba. No se observaron variaciones iguales o superiores en el resto de las pruebas. La Tabla 3 contiene el resumen de estos análisis.

Tabla 3. Puntaje pre y post de prueba TAE alumno y profesor en niños tímidos y disruptivos

\begin{tabular}{|l|l|c|c|c|c|}
\hline \multicolumn{2}{|l|}{ Señalamiento } & $\begin{array}{c}\text { TAE alumno } \\
\text { PRE }\end{array}$ & $\begin{array}{c}\text { TAE alumno } \\
\text { POST }\end{array}$ & $\begin{array}{c}\text { TAE profesor } \\
\text { PRE }\end{array}$ & $\begin{array}{c}\text { TAE profesor } \\
\text { POST }\end{array}$ \\
\hline \multirow{5}{*}{ Tímido } & Media & 59,50 & 70,25 & 43,50 & 66,75 \\
\cline { 2 - 6 } & $\mathrm{N}$ & 4 & 4 & 4 & 4 \\
\cline { 2 - 6 } & Desviación estándar & 14,457 & 12,945 & 4,203 & 16,500 \\
\hline \multirow{5}{*}{ Disruptivo } & Media & 54,80 & 52,67 & 51,00 & 68,80 \\
\cline { 2 - 7 } & $\mathrm{N}$ & 5 & 6 & 5 & 5 \\
\cline { 2 - 7 } & Desviación estándar & 7,014 & 5,715 & 3,464 & 12,091 \\
\hline
\end{tabular}




\begin{tabular}{|l|l|c|c|c|c|}
\hline \multirow{4}{*}{ Total } & Media & 56,89 & 59,70 & 47,67 & 67,89 \\
\cline { 2 - 6 } & $\mathrm{N}$ & 9 & 10 & 9 & 9 \\
\cline { 2 - 6 } & Desviación estándar & 10,446 & 12,508 & 5,315 & 13,280 \\
\hline
\end{tabular}

\subsection{ANÁLISIS CUALITATIVOS}

\subsubsection{Encuesta de satisfacción}

Los resultados de las encuestas de satisfacción indican que los docentes y estudiantes de ambos cursos experimentales valoraron positivamente la experiencia en el taller. En general, los niños reportaron que el taller era un espacio en el que podían expresarse de un modo que no podían en los espacios de clases tradicionales; los docentes también valoraron la oportunidad de trabajar con sus estudiantes de un modo diferente al que utilizaban tradicionalmente.

\subsubsection{Bitácoras}

Posterior al término del taller se realizó una revisión y análisis de las bitácoras con el objetivo de identificar algunos aspectos cualitativos que podrían haberse registrado en las sesiones. Este análisis permitió dar cuenta del progreso en cuanto a la calidad de las interacciones entre los estudiantes y también relevar situaciones particulares que son llamativas en el contexto del taller, que no necesariamente son medidas en las pruebas cuantitativas.

Una de estas situaciones tiene relación con el entusiasmo y compromiso de los estudiantes en las actividades del taller. Es posible que el hecho de sentirse autores de un producto que luego sería editado y publicado en un sitio web haya contribuido en la generación de esta actitud positiva que fue registrada entre las sesiones.

Un segundo aspecto que fue registrado en las bitácoras es el progreso que tuvieron algunos grupos en la resolución de los problemas al tiempo que los niveles de discusión se complejizaban, debido a que aumentaba el número de participantes. En las primeras sesiones, en las que las actividades eran principalmente en pareja, las duplas tuvieron que acordar entre sí las decisiones que tomarían respecto de las actividades que proponían, lo que en general podían lograr sin mayores dificultades; sin embargo, al momento de trabajar con el grupo completo, las discusiones necesariamente incluían a un grupo más amplio, lo que aumentaba la diversidad de opiniones y la dificultad de lograr acuerdo. En las primeras sesiones en las que tuvieron que trabajar por completo en grupo, los estudiantes se "trancaban" en discusiones referidas al progreso de la historia. En algunos de estos casos era necesaria la intervención de alguno de los integrantes del equipo investigador para lograr destrabar el conflicto a través de intervenciones relacionadas con lo socioemocional. En las sesiones finales del taller, los grupos se mostraban más cohesionados, lograban acuerdos y asignaban tareas en función de los intereses y habilidades de cada integrante.

Un tercer aspecto surgido a partir de los registros de las bitácoras es el progreso de algunos estudiantes en específico que al inicio del taller se mostraban poco participativos con el resto de su grupo. Uno de los estudiantes identificados como conflictivo por el 
profesor fue uno de los que mostró uno de los mayores progresos en términos cualitativos. En las primeras sesiones, este estudiante no lograba integrarse adecuadamente al trabajo con sus compañeros; sin embargo, en las últimas sesiones logró trabajar mejor con su grupo e incluso fue uno de los que leyó el cuento frente al curso.

\section{DISCUSIÓN}

La experiencia de escribir un cuento en forma colaborativa durante el taller puso en juego las distintas personalidades, estilos y expectativas de la historia de cada estudiante. La diversidad de opiniones sobre una construcción compartida generaba conflictos entre los niños, quienes no siempre tenían las herramientas para poder resolverlos. En algunos casos, el conflicto que surgía de la interacción podía ser resuelto por los estudiantes, pero en otros casos era necesaria la intervención de los investigadores para "destrancar" el conflicto visibilizando otras alternativas de resolución que intencionaban el aprendizaje socioemocional a partir del reconocimiento de las emociones propias y las de los otros.

En línea con lo anterior, estas intervenciones que utilizaban los investigadores promovían habilidades que han sido consideradas esenciales para la convivencia, como la escucha de las opiniones del otro y también la autorregulación. En el formato de cátedra tradicional de colegio se dificultan este tipo de interacciones entre los estudiantes, dado que las interacciones en general se dan entre el profesor y estudiante. El formato del taller propuso una estructura en la que las interacciones generalmente eran entre los estudiantes, quienes tenían que resolver los conflictos para avanzar en las actividades asignadas para cada sesión. Esta forma de trabajo requirió de una forma distinta de participación de los profesores, quienes debían trabajar contenidos relacionados con lo socioemocional cuando era necesario apoyar la resolución de los conflictos dentro de cada grupo.

Otro aspecto relevante del taller fue la experiencia de los grupos de sentirse autores de sus historias. Los niños se mostraron entusiasmados y comprometidos con la ejecución del taller, ya que tenían control sobre el producto que estaban construyendo. Además, era necesario que los estudiantes se vincularan cooperativamente para generar acuerdos que permitieran avanzar en la construcción del cuento. Estos dos componentes, el sentido de autoría y el vínculo cooperativo, permiten comprender la positiva valoración del taller por los estudiantes, ya que este proponía estructuras de participación que fomentaban interacciones positivas entre los alumnos que luego se traducían en un producto concreto y publicado en un sitio web.

Un aspecto que podría desarrollarse con más detalle en futuras investigaciones es el impacto de intervenciones psicoeducativas como esta para la promoción de autoestima en niños identificados como tímidos o disruptivos. Si bien el grupo de niños identificado como tímido obtuvo resultados iniciales promedio en las evaluaciones de autoestima, es llamativo el progreso que evidenciaron al término del taller. Es posible que en instancias de participación más pequeñas, como es el trabajo en grupo, estos niños utilicen mejores estrategias de interacción con sus pares que las que utilizan cuando deben interactuar en grupos más amplios, como el curso. Este uso de estrategias de interacción más efectivas puede traducirse en una mejora de la autoestima. En el caso de los niños identificados como disruptivos, es interesante ver que se produce un aumento en la autoestima percibida por los profesores, pero no por los alumnos. Es posible que el trabajo en grupos más pequeños 
haya permitido que este grupo de niños aprendiera estrategias de interacción que resultan menos disruptivas desde la mirada del profesor, a pesar de que ellos mismos no reporten un cambio en su autoestima.

\section{CONCLUSIONES}

Los resultados cuantitativos a nivel general indican que no es posible observar efectos de la intervención en el modo en que fue implementada. Las variaciones en puntaje que se observan entre las aplicaciones pre y post no se explican por los talleres dado que también se observan en el grupo control. Es posible que existan variables que no fueron controladas que explican los resultados en las aplicaciones post de ambos grupos.

A nivel específico, los resultados cuantitativos son consistentes con investigaciones previas (Humphrey et al., 2010) en la medida que son los grupos de niños que requieren de apoyos especiales los que muestran una mejora en intervenciones como esta.

Los resultados cualitativos dan cuenta de mejoras en la formas de interacción entre los estudiantes. Las dinámicas de trabajo observadas en el taller mostraron un progreso desde la primera sesión, en la que los estudiantes tenían dificultades para lograr acuerdos referidos a la construcción de la historia, hasta la última, en la que se mostraron cohesionados como grupo y dividían las tareas en función de los intereses y habilidades de cada uno.

\section{LIMITACIONES}

El contexto escolar impone restricciones importantes a estudios con diseños experimentales o cuasiexperimentales. Una de estas restricciones es que los estudiantes no pueden distribuirse aleatoriamente a las condiciones de intervención, ya que los grupos (cursos) se encuentran definidos de antemano. Esto puede introducir un sesgo de selección, lo que implica que los grupos pueden ser diferentes en características que son relevantes para la intervención, por ejemplo que uno de los grupos tenga mejores habilidades socioemocionales de base que el otro. Es por esto que se compararon los puntajes en una variedad de pruebas: comprensión lectora, alfabetización socioemocional, autoestima, clima escolar entre el grupo control y experimental para detectar posibles diferencias estadísticamente significativas en estas variables. Sin embargo, es posible que los grupos sean diferentes en otras variables, no registradas, y relevantes para los resultados.

\section{REFERENCIAS BIBLIOGRÁFICAS}

Agencia de Calidad de la Educación. (s/f). Otros Indicadores de Calidad Educativa. Recuperado de www.agenciaeducacion.cl/coordinacion-sac/otros-indicadores-de-calidad-educativa.

Alliende, A., Condemarín, M., \& Milicic, N. (1990). Prueba CLP formas paralelas. Santiago: Ediciones UC.

Antidote. (2003). The emotional literacy handbook. Promoting whole-school strategies. Gran Bretaña: David Fulton Publishers.

Arón, A., \& Milicic, N. (2013). Clima social escolar: escalas para su evaluación. México: Trillas. 
Arón, A., Milicic, N., \& Armijo, I. (2012). Clima Social Escolar: una escala de evaluación-Escala de Clima Social Escolar, ECLIS-. Universitas Psychologica, 11(3), 803-813.

Bailey, C. S., Denham, S. A., \& Curby, T. W. (2013). Early Child Development and Care Questioning as a component of scaffolding in predicting emotion knowledge in preschoolers. Early Child Development and Care, 183(2), 265-279. doi:10.1080/03004430.2012.671815

Berger, C., Álamos, P., Milicic, N., \& Alcalay, L. (2014). Rendimiento académico y las dimensiones personal y contextual del aprendizaje socioemocional: Evidencias de su asociación en estudiantes chilenos. Universitas Psychologica, 13(2), 627-638. doi:10.11144/Javeriana.UPSY13-2.radp

Brown, J., Jones, S., LaRusso, M., \& Aber, L. (2010). Improving Classroom Quality: Teacher Influences and Experimental Impacts of the 4Rs Program. Journal of Educational Psychology, 102(1), 153-167.

Colomer, T. (2012). La literatura que acoge: un proyecto de investigación en las aulas. En T. Colomer y M. Fittipaldi (Eds.), La literatura que acoge: inmigración y lectura de álbumes (pp. 7-25). Barcelona: Banco del Libro.

Delors, J. (Ed). (1997). La Educación encierra un tesoro. Informe a la UNESCO de la Comisión Internacional sobre la educación para el Siglo XXI. España: Santillana-UNESCO.

Durlak, J., Weissberg, R., Dymnicki, A., Taylor, R., \& Schellinger, K. (2011). The Impact of Enhancing Students' Social and Emotional Learning: A Meta-Analysis of School-Based Universal Interventions. Child Development, 82(1), 405-432. doi:10.1111/j.1467-8624.2010.01564.x

Dyer, J. R., Shatz, M., \& Wellman, H. M. (2000). Young children's storybooks as a source of mental state information. Cognitive Development, 15(1), 17-37. doi:10.1016/S0885-2014(00)00017-4

Hoffmann, D. (2009). Reflecting on social and emotional learning: A critical perspective on trends in the United States. Review of Educational Research, 79(2), 533-557. doi:10.3102/0034654308325184

Hruby, G.G, Goswami, U., Frederiksen, C.H., \& Perfetti, C.A. (2011). Neuroscience and Reading: A Review for Reading Education. Reading Research Quarterly, 46(2), 156-172. doi:10.1598/ RRQ.46.2.4

Humphrey, N. (2013). Social and emotional learning: a critical appraisal. London: Sage.

Humphrey, N., Kalambouka, A., Wigelsworth, M., \& Lendrum, A. (2010). Going for Goals: An Evaluation of a Short, Social-Emotional Intervention for Primary School Children. School Psychology International, 31(3), 250-270. doi:10.1177/0143034309352578

Jones, S. M., \& Bouffard, S. M. (2012). Social and Emotional Learning in Schools: From Programs to Strategies. Social Policy Report, 26(4), 3-33.

Marchant, T., Haeussler, I., \& Torreti, A. (2001). TAE Batería de tests de autoestima escolar (2a ed.). Santiago de Chile: Ediciones UC.

Morín, E. (1999). Los siete saberes necesarios para la educación del futuro. España: SantillanaUNESCO.

Ripley, K., \& Simpson, E. (2007). First steps to emotional literacy. Estados Unidos: Routledge.

Riquelme, E., \& Munita, F. (2011). La lectura mediada de literatura infantil como herramienta para la alfabetización emocional. Estudios Pedagógicos, 37(1), 269-277.

Sanjuán, M. (2014). La dimensión emocional en la educación literaria. España: Prensas de la Universidad de Zaragoza.

Santagostino, P. (1997). Cómo contar un cuento e inventarse cientos. Barcelona: Ediciones Obelisco. Shonkoff, J. P., \& Philips, D. A. (2000). From Neurons to Neighborhoods. The Science of Early Childhood Development. Washington, DC: National Academy Press.

Thompson, R.A. (2010). Feeling and understanding through the prism of relationships. En S.D., Calkins \& M.A. Bell (Eds.), Child development at the intersection of emotion and cognition (pp. 79-95). Washington, D.C: APA. 
